\title{
2.
}

\section{ON THE PROPERTIES OF A CERTAIN SYMBOLICAL EXPRESSION.}

[From the Cambridge Mathematical Journal, vol, III. (1841), pp. 62-71.]

THE series

$$
\begin{gathered}
S_{p 0}^{\infty} \cdot \zeta_{p}\left(a^{2}+b^{2} \ldots \mathfrak{n} \text { terms }\right)^{p+i}\left(\frac{l}{1+l} \cdot \frac{d^{2}}{d a^{2}}+\frac{m}{1+m} \cdot \frac{d^{2}}{d b^{2}} \ldots\right)^{p} \frac{1}{\left\{(1+l) a^{2}+(1+m) b^{2} \ldots\right\}^{i}} \\
\left(\zeta_{p}=\frac{1}{2^{2 p+1} 1.2 \ldots p \cdot i(i+1) \ldots(i+p)}\right) \ldots \ldots(\psi),
\end{gathered}
$$

possesses some remarkable properties, which it is the object of the present paper to investigate. We shall prove that the symbolical expression $(\psi)$ is independent of $a, b$, \&c., and equivalent to the definite integral

$$
\int_{0}^{1} \frac{x^{2 i-1} d x}{\left\{\left(1+l x^{2}\right)\left(1+m x^{2}\right) \ldots\right\}^{\frac{1}{2}}}
$$

a property which we shall afterwards apply to the investigation of the attractions of an ellipsoid upon an external point, and to some other analogous integrals. The demonstration of this, which is one of considerable complexity, may be effected as follows :

Writing the symbol $\frac{l}{1+l} \cdot \frac{d^{2}}{d a^{2}}+\frac{m}{1+m} \cdot \frac{d^{2}}{d b^{2}} \ldots$ under the form

$$
\left(\frac{d^{2}}{d a^{2}}+\frac{d^{2}}{d b^{2}} \ldots\right)-\left(\frac{1}{1+l} \cdot \frac{d^{2}}{d a^{2}}+\frac{1}{1+m} \cdot \frac{d^{2}}{d b^{2}} \ldots\right)=\Delta-\left(\frac{1}{1+l} \cdot \frac{d^{2}}{d a^{2}}+\frac{1}{1+m} \cdot \frac{d^{2}}{d b^{2}} \ldots\right) \text { suppose, }
$$

let the $p^{\text {th }}$ power of this quantity be expanded in powers of $\Delta$. The general term is

$$
(-1)^{q} \cdot \frac{p(p-1) \ldots(p-q+1)}{1.2 \ldots q} \cdot \Delta^{p-q}\left(\frac{1}{1+l} \cdot \frac{d^{2}}{d a^{2}} \ldots\right)^{q},
$$

which is to be applied to

$$
\frac{1}{\left\{(1+l) a^{2} \ldots\right\}^{i}} \text {. }
$$


Considering the expression

if for a moment we write

$$
\left(\frac{1}{1+l} \cdot \frac{d^{2}}{d a^{2}} \ldots\right)^{q} \frac{1}{\left\{(1+l) a^{2} \cdots\right\}^{i}}
$$

$$
(1+l) a^{2}=a_{1}^{2}, \& \text { c.; } \quad \Delta_{1}=\frac{d^{2}}{d a_{1}{ }^{2}}+\frac{d^{2}}{d b_{1}{ }^{2}} \ldots ; \quad \rho_{1}=a_{1}{ }^{2}+b_{1}{ }^{2} \ldots,
$$

this becomes

$$
\Delta_{1}^{q} \frac{1}{\rho_{1}^{i}} \text {. }
$$

Now it is immediately seen that $\Delta_{1} \frac{1}{\rho_{1}^{i^{\prime}}}=\frac{2 i^{\prime}\left(2 i^{\prime}+2-\mathfrak{n}\right)}{\rho_{1}^{i+1}}$;

from which we may deduce

$$
\Delta_{1}^{q} \frac{1}{\rho_{1}^{i}}=\frac{2 i(2 i+2) \ldots(2 i+2 q-2)(2 i+2-\mathfrak{n}) \ldots(2 i+2 q-\mathfrak{n})}{\rho_{1}^{i+q}},
$$

or, restoring the value of $\rho_{1}$, and forming the expression for the general term of $(\psi)$, this is

$$
\zeta_{p} \cdot \rho^{p+1}\left\{\begin{array}{c}
\Delta^{p} \frac{1}{\left(a^{2}+b^{2} \ldots+l a^{2}+m b^{2}+\& c .\right)^{i}} \\
-\frac{p}{1} 2 i(2 i+2-\mathfrak{n}) \Delta^{p-1} \frac{1}{\left(a^{2}+b^{2}+\ldots+l a^{2}+m b^{2}+\ldots\right)^{i}} \\
+\& \mathrm{c} .
\end{array}\right.
$$

$\rho$ representing the quantity $a^{2}+b^{2}+\& c$.

Hence, selecting the terms of the $s^{\text {th }}$ order in $l, m$, \&c. the expression for the part of $(\psi)$ which is of the $s^{\text {th }}$ order in $l, m$ \&c. may be written under the form

multiplied by

$$
S_{p_{0}}^{s} \frac{(-1)^{8} \rho^{p+1} \zeta_{p}}{1.2 \ldots s}
$$

$$
\left\{\begin{array}{c}
i(i+1) \ldots(i+s-1) \Delta^{p} \frac{U}{\rho^{i+s}} \\
-\frac{p}{1} 2 i(2 i+2-\mathfrak{n})(i+1) \ldots \quad(i+s) \Delta^{p-1} \frac{U}{\rho^{i+8+1}} \\
+\frac{p(p-1)}{1.2} 2 i(2 i+2)(2 i+2-\mathfrak{n})(2 i+4-\mathfrak{n})(i+2) \ldots(i+s+1) \Delta^{p-2} \frac{U}{\rho^{i+8+2}} \\
-\& c . \quad\left[l a^{2}+m b^{2} \ldots=U \text { suppose }\right]
\end{array}\right.
$$

which for conciseness we shall represent by

$=S$ suppose.

$$
\frac{(-1)^{s}}{1.2 \ldots s} S_{p 0}^{s} \rho^{p+1} \cdot \zeta_{p}\left\{\begin{array}{c}
\alpha_{s} \Delta^{p} \frac{U}{\rho^{i+8}} \\
-\frac{p}{1} \beta_{s} \Delta^{p-1} \frac{U}{\rho^{i+8+1}} \\
+\frac{p(p-1)}{1.2} \gamma_{s} \Delta^{p-2} \frac{U}{\rho^{i+8+2}} \\
-\& c .
\end{array}\right.
$$


Now $U$ representing any homogeneous function of the order $2 s$, it is easily seen that

$$
\Delta \frac{U}{\rho^{i}}=\frac{\Delta U}{\rho^{i}}+2 i(2 i+2-4 s-\mathfrak{m}) \frac{U}{\rho^{i+1}} ;
$$

and repeating continually the operation $\Delta$, observing that $\Delta U, \Delta^{2} U$, \&c. are of the orders $2(s-1), 2(s-2)$, \&c. we at length arrive at

$$
\begin{aligned}
\Delta^{q} \cdot \frac{U}{\rho^{i}}= & \Delta^{q} U \cdot \frac{1}{\rho^{i}} \\
& +\frac{q}{1} 2 i(2 i+2 q-4 s-\mathfrak{n}) \Delta^{q-1} U \cdot \frac{1}{\rho^{i+1}} \\
+ & \frac{q(q-1)}{1.2} 2 i(2 i+2)(2 i+2 q-4 s-\mathfrak{n})(2 i+2 q-4 s-\mathfrak{n}-2) \Delta^{q-2} U \cdot \frac{1}{\rho^{i+2}} \\
\vdots & \quad+2 i(2 i+2) \ldots(2 i+2 q)(2 i+2 q-4 s-\mathfrak{n}) \ldots \quad(2 i+2-4 s-\mathfrak{x}) U \cdot \frac{1}{\rho^{i+q}} .
\end{aligned}
$$

Changing $i$ into $s+i+i^{\prime}$, we have an equation which we may represent by

$$
\Delta^{q} \frac{U}{\rho^{s+i+i^{\prime}}}=A_{q}, i^{\prime} \frac{\Delta^{q} U}{\rho^{s+i+i^{\prime}}}+{ }^{1} A_{q}, i^{\prime} \rho^{\frac{\Delta^{q-1} U}{8+i+i^{\prime}+1}} \ldots+{ }^{q} A_{q}, i^{\prime} \frac{U}{\rho^{8+i+i^{\prime}+q}} \cdots
$$

where in general

$$
\begin{aligned}
& { }^{r} A_{q, i^{\prime}}=\frac{q(q-1) \ldots(q-r+1)}{1.2 \ldots r} \\
& \quad \times\left(2 s+2 i+2 i^{\prime}\right)\left(2 s+2 i^{\prime}+2 i+2\right) \ldots\left(2 s+2 i^{\prime}+2 i+2 r-2\right) \\
& \quad \times\left(2 i+2 i^{\prime}+2 q-2 s-\mathfrak{n}\right) \ldots\left(2 i+2 i^{\prime}+2 q-2 s-\mathfrak{n}-2 r+2\right) .
\end{aligned}
$$

Now the value of $S$, written at full length, is

$$
\frac{(-1)^{s}}{1.2 \ldots s}\left\{\begin{aligned}
& \zeta_{s} \rho^{s+1}\left(\alpha_{s} \Delta^{s} \frac{U}{\rho^{s+1}}-\frac{s}{1} \beta_{s} \Delta^{s-1} \frac{U}{\rho^{s+i+1}} \ldots\right. \\
+ & \zeta_{s-1} \rho^{s+i-1}\left(\alpha_{s} \Delta^{s-1} \frac{U}{\rho^{s+1}}-\frac{s-1}{1} \beta_{s} \Delta^{s-2} \frac{U}{\rho^{s+i+1}}+\ldots\right. \\
+ & \& \mathrm{c} .
\end{aligned}\right.
$$

and substituting for the several terms of this expansion the values given by the equation $(\alpha)$, we have

where in general

$$
S=\frac{(-1)^{s}}{1.2 \ldots \ldots s}\left(k_{0} \Delta^{s} U+k_{1} \frac{1}{\rho} \Delta^{s-1} U \ldots \ldots+k_{s} \frac{1}{\rho^{8}} U\right)
$$

$$
\begin{aligned}
k_{\mathrm{x}} & =\alpha_{s}\left({ }^{\mathrm{x}} A_{s}, 0 \zeta_{s}+{ }^{\mathrm{x}-1} A_{s-1}, 0 \zeta_{s-1} \ldots+A_{s-\mathrm{x}, 0} \zeta_{s-\mathrm{x}}\right) \\
& -\beta_{s}\left(\frac{s}{1}{ }^{\mathrm{x}-1} A_{s-1}, 1 \zeta_{s} \ldots+\frac{(s-\mathrm{x}+1)}{1} A_{s-\mathrm{x}, 1} \zeta_{s-\mathrm{x}+1}\right) \\
& \vdots \\
& \pm \lambda_{s}\left(\frac{s(s-1) \ldots(s-\mathrm{x}+1)}{1.2 \ldots \mathrm{x}} A_{s-\mathrm{x}, \mathrm{x}} \zeta_{s}\right),
\end{aligned}
$$

$\lambda_{s}$ being the $(x+1)^{\text {th }}$ of the series $\alpha_{s}, \beta_{s} \ldots$ 
Substituting for the quantities involved in this expression, and putting, for simplicity $2 i+2-\mathfrak{n}=2 \gamma$, we have, without any further reduction, except that of arranging the factors of the different terms, and cancelling those which appear in the numerator and denominator of the same term,

$$
\frac{(-1)^{s} k_{\mathrm{x}}}{1.2 \ldots \ldots s}=\frac{(-1)^{s-\mathrm{x}}(1-\gamma)(2-\gamma) \ldots(\mathrm{x}-\gamma)}{2^{2 s+1} \cdot 1.2 \ldots s \cdot 1.2 \ldots(s-\mathrm{x}) \cdot 1 \cdot 2 \ldots \mathrm{x}}
$$

multiplied by the series

$$
\begin{aligned}
& \begin{array}{l}
(i+s+1) \\
\ldots(i+s+\mathrm{x}-1) \text { into }
\end{array} \\
& \quad\left\{1+\frac{\gamma}{1} \frac{\mathrm{x}}{\mathrm{x}-\gamma}+\frac{\gamma(\gamma+1)}{1.2} \frac{\mathrm{x}(\mathrm{x}-1)}{(\mathrm{x}-\gamma)(\mathrm{x}-1-\gamma)}+\ldots \quad(\mathrm{x}+1) \text { terms }\right\} \\
& -\frac{(i+s) \ldots(i+s+\mathrm{x}-2)}{1-\gamma} \text { into } \\
& \quad\left\{\begin{array}{l}
\mathrm{x}+\frac{\gamma}{1} \frac{\mathrm{x}(\mathrm{x}-1)}{\mathrm{x}-\gamma}+\frac{\gamma(\gamma+1)}{1.2} \frac{\mathrm{x}(\mathrm{x}-1)(\mathrm{x}-2)}{(\mathrm{x}-\gamma)(\mathrm{x}-1-\gamma)}+\ldots \\
\left.+(-1)^{r} \frac{(i+s-r+1) \ldots(i+s+\mathrm{x}-r-1)}{(1-\gamma)(2-\gamma) \ldots(r-\gamma)} \text { into terms }\right\} \\
\left\{\mathrm{x}(\mathrm{x}-1) \ldots(\mathrm{x}-r+1)+\frac{\gamma}{1} \frac{\mathrm{x}(\mathrm{x}-1) \ldots(\mathrm{x}-r)}{\mathrm{x}-\gamma}+\ldots(\mathrm{x}+r-1) \text { terms }\right\}
\end{array}\right.
\end{aligned}
$$

to $r=\mathrm{x}$.

Now it may be shown that

$$
\begin{aligned}
& \frac{1}{(1-\gamma)(2-\gamma) \ldots(r-\gamma)} \\
& \left\{x(x-1) \ldots(x-r+1)+\frac{\gamma}{1} \frac{x(x-1) \ldots(x-r)}{x-\gamma}+\& c \ldots(x+1-r) \text { terms }\right\} \\
& \quad=\frac{x(x-1) \ldots(r+1) \cdot x(x-1) \ldots(x-r+1)}{(1-\gamma)(2-\gamma) \ldots(x-\gamma)}
\end{aligned}
$$

which reduces the expression for $k_{\mathrm{x}}$ to the form

$$
\frac{(-1)^{8} k_{\mathrm{x}}}{1.2 \ldots s}=\frac{(-1)^{s+\mathrm{x}}}{2^{2 s+1} \cdot 1.2 \ldots s .1 .2 \ldots(s-\mathrm{x})}\left\{\begin{array}{rr}
(i+s+1) \ldots(i+s+\mathrm{x}-1) \\
-\frac{\mathrm{x}}{1}(i+s) \ldots & (i+s+\mathrm{x}-2) \\
+\frac{\mathrm{x}(\mathrm{x}-1)}{1.2}(i+s-1) \ldots(i+s+\mathrm{x}-3) \\
\pm \& \mathrm{c} .(\mathrm{x}+1) \text { terms }
\end{array}\right.
$$

from which it may be shown, that except for $\mathrm{x}=0, k_{\mathrm{x}}=0$.

The value $\mathrm{x}=0$, observing that the expression

$$
(i+s+1)(i+s+2) \ldots(i+s-1)
$$

represents $\frac{1}{i+s}$, gives 


$$
\frac{(-1)^{s} k_{0}}{1.2 \ldots s}=\frac{(-1)^{s}}{2^{2 s}(1.2 \ldots s)^{2} \cdot(2 i+2 s)}
$$

or we have simply

where

$$
\begin{aligned}
& S=\frac{(-1)^{s}}{2^{2 s}(1.2 \ldots s)^{2} \cdot(2 i+2 s)} \Delta^{s} U \\
& \Delta=\frac{d^{2}}{d a^{2}}+\frac{d^{2}}{d b^{2}}+\ldots, \quad U=\left(l a^{2}+m b^{2} \ldots\right)^{s} .
\end{aligned}
$$

Consider the term

$$
\frac{1.2 \ldots s}{1.2 \ldots \lambda .1 .2 \ldots \mu . \& \mathrm{c} .} a^{2 \lambda} b^{2 \mu} \ldots l^{\lambda} m^{\mu} \ldots ;
$$

with respect to this, $\Delta^{s}$ reduces itself to

$$
\frac{1.2 \ldots s}{1.2 \ldots \lambda .1 .2 \ldots \mu . \& c .}\left(\frac{d}{d a}\right)^{2 \lambda} \ldots
$$

and the corresponding term of $S$ is

$$
\begin{gathered}
\frac{(-1)^{s}}{2^{2 s}(2 i+2 s)(1.2 \ldots \lambda .1 .2 \ldots \mu . \& \mathrm{c} .)^{2}} 1.2 \ldots 2 \lambda .1 .2 \ldots 2 \mu . \& \mathrm{c} . l^{\lambda} m^{\mu} \ldots \\
=\frac{(-1)^{s} \cdot 1.3 \ldots(2 \lambda-1) \cdot 1.3 \ldots(2 \mu-1) . \& \mathrm{c} .}{(2 i+2 s) 2.4 \ldots 2 \lambda .2 .4 \ldots 2 \mu . \& \mathrm{c} .} l^{\lambda} m^{\mu} \ldots
\end{gathered}
$$

which, omitting the factor $\frac{1}{2 i+2 s}$, and multiplying by $x^{2 s}$, is the general term of the $s^{\text {th }}$ order in $l, m, \ldots$ of

$$
\frac{1}{\sqrt{\left\{\left(1+l x^{2}\right)\left(1+m x^{2}\right) \ldots\right\}}} \text {. }
$$

The term itself is therefore the general term of

$$
\int_{0}^{1} \frac{x^{2 i-1} d x}{\sqrt{\left\{\left(1+l x^{2}\right)\left(1+m x^{2}\right) \ldots\right\}}}
$$

or taking the sum of all such terms for the complete value of $S$, and the sum of the different values of $S$ for the values $0,1,2 \ldots$ of the variable $s$, we have the required equation

$$
\psi=\int_{0}^{1} \frac{x^{2 i-1} d x}{\sqrt{\left\{\left(1+l x^{2}\right)\left(1+m x^{2}\right) \ldots\right\}}}
$$

Another and perhaps more remarkable form of this equation may be deduced by writing $\frac{a^{2}}{1+l}, \frac{b^{2}}{1+m}$, \&c. for $a^{2}, b^{2}$, \&c., and putting $\frac{a^{2}}{1+l}+\frac{b^{2}}{1+m}+\& c .=\eta^{2}, l \eta^{2}=a^{2}$, $m \eta^{2}=\beta^{2}$, \&c.: we readily deduce

$$
\begin{gathered}
\eta^{\mathfrak{n}-2 i} \int_{0}^{1} \frac{x^{2 i-1} d x}{\sqrt{\left\{\left(\eta^{2}+\alpha^{2} x^{2}\right)\left(\eta^{2}+\beta^{2} x^{2}\right) \ldots\right\}}} \\
=\boldsymbol{S}_{p \circ}^{\infty} \frac{1}{2^{2 p+1} \cdot 1.2 \ldots p \cdot i(i+1) \ldots(i+p)}\left(\alpha^{2} \frac{d^{2}}{d a^{2}}+\beta^{2} \frac{d^{2}}{d b^{2}} \ldots\right)^{p} \frac{1}{\left(a^{2}+b^{2} \ldots\right)^{i}},
\end{gathered}
$$

c. 
$\eta$ being determined by the equation

$$
\frac{a^{2}}{\eta^{2}+\alpha^{2}}+\frac{b^{2}}{\eta^{2}+\beta^{2}} \ldots=1
$$

or, as it may otherwise be written,

$$
\eta^{2}=a^{2}+b^{2}+\ldots-\frac{a^{2} \alpha^{2}}{\eta^{2}+\alpha^{2}}-\frac{b^{2} \beta^{2}}{\eta^{2}+\beta^{2}}-\& c .
$$

$\mathrm{n}$, it will be recollected, denotes the number of the quantities $a, b$, \&c.

Now suppose

$$
V=\iint \ldots \phi(a-x, b-y, \ldots) d x d y \ldots
$$

(the integral sign being repeated $\mathfrak{n}$ times) where the limits of the integral are given by the equation

$$
\frac{x^{2}}{h^{2}}+\frac{y^{2}}{h_{1}^{2}}+\& c .=1
$$

and that it is permitted, throughout the integral to expand the function $\phi(a-x, \ldots)$ in ascending powers of $x, y$, \&c. (the condition for which is apparently that of $\phi$ not becoming infinite for any values of $x, y$, \&c., included within the limits of the integration): then observing that any integral of the form $\iint \ldots x^{p} y^{q} \ldots d x d y \& c . .$. where any one of the exponents $p, q, \& c . .$. is odd, when taken between the required limits contains equal positive and negative elements and therefore vanishes, the general term of $V$ assumes the form

$$
\frac{1}{1.2 \ldots 2 r .1 .2 \ldots 2 s \ldots}\left(\frac{d}{d a}\right)^{2 r}\left(\frac{d}{d b}\right)^{2 s} \ldots \phi(a, b \ldots) \iint \ldots x^{2 r} y^{2 s} \ldots d x d y \ldots
$$

Also, by a formula quoted in the eleventh No. of the Mathematical Journal, the value of the definite integral $\iint \ldots x^{2 r} y^{28} \ldots d x d y \ldots$ is

$$
h^{2 r+1} h_{r}{ }^{2 s+1} \ldots \frac{\Gamma\left(r+\frac{1}{2}\right) \Gamma\left(s+\frac{1}{2}\right) \ldots}{\Gamma\left(r+s+\ldots+\frac{1}{2} \mathfrak{n}+1\right)},
$$

(observing that the value there given referring to positive values only of the variables, must be multiplied by $\left.2^{\mathfrak{k}}\right)$ : or, as it may be written

$$
h^{2 r+1} h_{,}^{2 s+1} \ldots \pi^{\frac{1}{2} \mathfrak{n}} \cdot \frac{1}{2^{r+8} \ldots \frac{1}{2} \mathfrak{n}\left(\frac{1}{2} \mathfrak{n}+1\right) \ldots\left(\frac{1}{2} \mathfrak{n}+r+s \ldots\right) \Gamma\left(\frac{1}{2} \mathfrak{n}\right)}:
$$

hence the general term of $V$ takes the form

$$
\begin{aligned}
& \frac{h h, \ldots \pi^{\frac{1}{2} \mathfrak{n}}}{\Gamma\left(\frac{1}{2} \mathfrak{n}\right)} \frac{1}{\frac{1}{2} \mathfrak{n}\left(\frac{1}{2} \mathfrak{n}+1\right) \ldots\left(\frac{1}{2} \mathfrak{n}+r+s \ldots\right)} \cdot \frac{1}{2^{2 r+28 \ldots}} \frac{1}{1.2 .3 \ldots r .1 .2 \ldots s \ldots} \\
& \times\left(h^{2} \frac{d^{2}}{d a^{2}}\right)^{r}\left(h^{2} \frac{d^{2}}{d b^{2}}\right)^{s} \ldots \phi(a, b, \ldots) ;
\end{aligned}
$$

and putting $r+s+\& c .=p$, and taking the sum of the terms that answer to the same value of $p$, it is immediately seen that this sum is

$$
=\frac{h h, \ldots \pi^{\frac{1}{2} \mathfrak{n}}}{\Gamma\left(\frac{1}{2} \mathfrak{n}\right)} \cdot \frac{1}{2^{2 p} \cdot 1.2 \ldots p \cdot \frac{1}{2} \mathfrak{n}\left(\frac{1}{2} \mathfrak{n}+1\right) \ldots\left(\frac{1}{2} \mathfrak{n}+p\right)}\left(h^{2} \frac{d^{2}}{d a^{2}}+h_{,}^{2} \frac{d^{2}}{d b^{2}} \ldots\right)^{p} \phi(a, b \ldots) .
$$


Or the function $\phi(a-x, b-y \ldots)$ not becoming infinite within the limits of the integration, we have

$$
\iint \ldots \phi(a-x, b-y \ldots) d x d y \ldots
$$

$=\frac{2 h h, \ldots \pi^{\frac{1}{3} \mathfrak{n}}}{\Gamma\left(\frac{1}{2} \mathfrak{n}\right)} \boldsymbol{S}_{p 0}^{\infty} \frac{1}{2^{2 p+1} \cdot 1.2 \ldots p \cdot \frac{1}{2} \mathfrak{n}\left(\frac{1}{2} \mathfrak{n}+1\right) \ldots\left(\frac{1}{2} \mathfrak{n}+p\right)}\left(h^{2} \frac{d^{2}}{d a^{2}}+h_{1}{ }^{2} \frac{d^{2}}{d b^{2}} \ldots\right)^{p} \phi(a, b \ldots)$

the integral on the first side of the equation extending to all real values of $x, y$, \&c., subject to $\frac{x^{2}}{h^{2}}+\frac{y^{2}}{h^{2}}+\ldots<1$.

Suppose in the first place $\quad \phi(a, b \ldots)=\frac{1}{\left(a^{2}+b^{2} \ldots\right)^{\frac{1}{2} n}}$.

By a preceding formula the second side of the equation reduces itself to

$$
\begin{gathered}
\frac{2 h h, \ldots \pi^{\frac{1}{2} \mathfrak{n}}}{\Gamma\left(\frac{1}{2} \mathfrak{n}\right)} \int_{0}^{1} \frac{x^{\mathfrak{n}-1} d x}{\sqrt{\left\{\left(\eta^{2}+h^{2} x^{2}\right)\left(\eta^{2}+h_{\imath}^{2} x^{2}\right) \ldots(\mathfrak{n} \text { factors })\right\}}} \\
\frac{a^{2}}{\eta^{2}+h^{2}}+\frac{b^{2}}{\eta^{2}+h_{\imath}^{2}} \ldots=1
\end{gathered}
$$

$\eta$ being given by

Hence the formula

$$
\begin{aligned}
& \iint \ldots n \text { times } \frac{d x d y \ldots}{\left\{(a-x)^{2}+(b-y)^{2} \ldots\right\}^{\frac{1}{2} n}}
\end{aligned}
$$

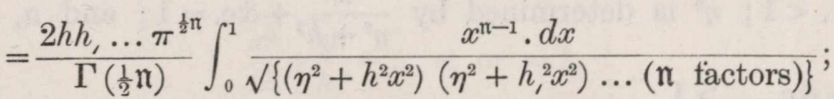

where the integral on the first side of the equation extends to all real values of $x, y$, \&c. satisfying $\frac{x^{2}}{h^{2}}+\frac{y^{2}}{h_{1}^{2}}+\&$ c. $\ldots<1 ; \eta^{2}$, as we have seen, is determined by

$$
\frac{a^{2}}{\eta^{2}+h^{2}}+\frac{b^{2}}{\eta^{2}+h_{,}^{2}}+\& c .=1
$$

and finally, the condition of $\phi(a-x, b-y \ldots)$ not becoming infinite within the limits of the integration, reduces itself to $\frac{a^{2}}{h^{2}}+\frac{b^{2}}{h_{\imath}{ }^{2}}+\ldots>1$, which must be satisfied by these quantities.

Suppose in the next place that the function $\phi(a, b \ldots)$ satisfies $\frac{d^{2} \phi}{d a^{2}}+\frac{d^{2} \phi}{d b^{2}}+\& c .=0$

The factor $\left(h^{2} \frac{d^{2}}{d a^{2}}+\& c.\right)$ may be written under the form

$$
\left(h_{\prime}^{2}-h^{2}\right) \frac{d^{2}}{d b^{2}}+\left(h_{\prime \prime}{ }^{2}-h^{2}\right) \frac{d^{2}}{d c^{2}}+\& c .+h^{2}\left(\frac{d^{2}}{d a^{2}}+\frac{d^{2}}{d b^{2}} \ldots\right)=\left(h_{,}^{2}-h^{2}\right) \frac{d^{2}}{d b^{2}}+\& c .
$$


since, as applied to the function $\phi, \frac{d^{2}}{d a^{2}}+\frac{d^{2}}{d b^{2}}+\& c$. is equivalent to 0 ; we have in this case

$$
\begin{aligned}
& \iint \ldots \phi(a-x, b-y \ldots) d x d y \ldots \\
& =\frac{2 h h_{1} \ldots \pi^{3 \mathfrak{n}}}{\Gamma\left(\frac{1}{2} \mathfrak{n}\right)} \mathbb{S}_{p \circ}^{\infty} \frac{1}{2^{2 p+1} \cdot 1.2 \ldots p \cdot \frac{1}{2} \mathfrak{n} \ldots\left(\frac{1}{2} \mathfrak{n}+p\right)}\left\{\left(h_{1}^{2}-h^{2}\right) \frac{d^{2}}{d b^{2}}+\ldots\right\}^{p} \phi(a, b \ldots) ;
\end{aligned}
$$

or the first side divided by $h h, \ldots$ has the remarkable property of depending on the differences $h_{l}^{2}-h^{2}$, \&c. only; this is the generalisation of a well-known property of the function $V$, in the theory of the attraction of a spheroid upon an external point.

If in this equation we put $\phi(a, b \ldots)=\frac{a}{\left(a^{2}+b^{2} \ldots\right)^{3 n}}$, which satisfies the required condition $\frac{d^{2} \phi}{d a^{2}}+\& c .=0$, then transferring the factor $a$ to the left-hand side of the sign $S$, and putting in a preceding formula, $a^{2}=0, \beta^{2}=h_{,}^{2}-h^{2}$, \&c. and $\eta^{2}+h^{2}$ for $\eta^{2}$, we obtain

$$
\begin{aligned}
& \iint \ldots(\mathfrak{n} \text { times }) \frac{(a-x) d x d y \ldots}{\left\{(a-x)^{2}+(b-y)^{2} \ldots\right\}^{\mathfrak{l n}^{\mathfrak{n}}}} \\
& =\frac{2 h h_{1} \ldots \pi^{\mathfrak{3} \mathfrak{n}} a}{\sqrt{ }\left(\eta^{2}+h^{2}\right) \cdot \Gamma\left(\frac{1}{2} \mathfrak{n}\right)} \int_{0}^{1} \frac{x^{\mathfrak{n}-1} d x}{\sqrt{ }\left[\left\{\eta^{2}+h^{2}+\left(h_{\prime}{ }^{2}-h^{2}\right) x^{2}\right\}\left\{\eta^{2}+h^{2}+\left(h_{\prime \prime}{ }^{2}-h^{2}\right) x^{2}\right\} \ldots(\mathfrak{n}-1) \text { factors }\right]} \text {, }
\end{aligned}
$$

where, as before, the integrations on the first side extend to all real values of $x, y$, \&c., satisfying $\frac{x^{2}}{h^{2}}+\frac{y^{2}}{h_{l}^{2}} \ldots<1 ; \eta^{2}$ is determined by $\frac{a^{2}}{\eta^{2}+h^{2}}+\& c .=1$; and $a, b, \ldots h, h_{l}$, \&c. are subject to $\frac{a^{2}}{h^{2}}+\frac{b^{2}}{h_{1}^{2}}+\&$ c. ... $>1$.

For $\mathfrak{n}=3$, this becomes,

$$
\begin{gathered}
\iiint \frac{(a-x) d x d y d z}{\left\{(a-x)^{2}+(b-y)^{2}+(c-z)^{2}\right\}^{\frac{3}{2}}} \\
=\frac{4 \pi h h_{,} h_{l \prime} a}{\sqrt{\left(h^{2}+\eta^{2}\right)}} \int_{0}^{1} \frac{x^{2} d x}{\sqrt{\left[\left\{\eta^{2}+h^{2}+\left(h_{\prime}{ }^{2}-h^{2}\right) x^{2}\right\}\left\{\eta^{2}+h^{2}+\left(h_{\prime \prime}{ }^{2}-h^{2}\right) x^{2}\right\}\right]}}
\end{gathered}
$$

the integrations on the first side extending over the ellipsoid whose semiaxes are $h, h_{l}, h_{\mu}$, and the point whose coordinates are $a, b, c$, being exterior to this ellipsoid; also $\frac{a^{2}}{\eta^{2}+h^{2}}+\frac{b^{2}}{\eta^{2}+h_{\prime}{ }^{2}}+\frac{c^{2}}{\eta^{2}+h_{\prime \prime}{ }^{2}}=1$ : a known theorem. 